\title{
Review
}

\section{Anticancer strategies based on the metabolic profile of tumor cells: therapeutic targeting of the Warburg effect}

\author{
Xi-sha CHEN ${ }^{1, \#}$, Lan-ya LI" \#, Yi-di GUAN ${ }^{1}$, Jin-ming YANG ${ }^{2}$, Yan CHENG ${ }^{1, *}$ \\ ${ }^{1}$ Department of Pharmacology, School of Pharmaceutical Sciences, Central South University, Changsha 410008, China; ${ }^{2}$ Department \\ of Pharmacology, the Penn State Hershey Cancer Institute, the Pennsylvania State University College of Medicine and Milton S Hershey \\ Medical Center, Hershey, PA 17033, USA
}

Tumor cells rely mainly on glycolysis for energy production even in the presence of sufficient oxygen, a phenomenon termed the Warburg effect, which is the most outstanding characteristic of energy metabolism in cancer cells. This metabolic adaptation is believed to be critical for tumor cell growth and proliferation, and a number of onco-proteins and tumor suppressors, including the $\mathrm{PI3K} / \mathrm{Akt} / \mathrm{mTOR}$ signaling pathway, Myc, hypoxia-inducible factor and p53, are involved in the regulation of this metabolic adaptation. Moreover, glycolytic cancer cells are often invasive and impervious to therapeutic intervention. Thus, altered energy metabolism is now appreciated as a hallmark of cancer and a promising target for cancer treatment. A better understanding of the biology and the regulatory mechanisms of aerobic glycolysis has the potential to facilitate the development of glycolysis-based therapeutic interventions for cancer. In addition, glycolysis inhibition combined with DNA damaging drugs or chemotherapeutic agents may be effective anticancer strategies through weakening cell damage repair capacity and enhancing drug cytotoxicity.

Keywords: glycolysis cancer cells; cancer cells metabolism; Warburg effect; anticancer strategy

Acta Pharmacologica Sinica (2016) 37: 1013-1019; doi: 10.1038/aps.2016.47; published online 4 Jul 2016

\section{The Warburg effect-a hallmark of cancer}

By definition, cancer involves the inappropriate proliferation of cells, and cancer cells have the ability to cope with metabolic stress by enhancing alternative energy production mechanisms. Energy metabolism in most cancer cells differs remarkably from that of normal cells, and the Warburg effect represents one of the hallmarks of cancer ${ }^{[1,2]}$. Under aerobic conditions, normal cells rely on mitochondrial oxidative phosphorylation, a process that converts glucose to pyruvate in the cytosol, and thereafter to carbon dioxide in the mitochondria, producing the energy-storing molecule ATP. Under anaerobic conditions, glycolysis is favored, and pyruvate is converted to lactate in the cytosol. Most malignant cells mainly depend on glycolysis, the anaerobic metabolism of glucose producing ATP, even in the presence of sufficient oxygen, which leads to a state that has been termed "aerobic glycolysis" ${ }^{[3]}$. There are 36 molecules of ATP generated upon complete oxidation of

\footnotetext{
\# These authors contributed equally to this work.

${ }^{*}$ To whom correspondence should be addressed.

E-mail chengyan0677@163.com

Received 2016-02-14 Accepted 2016-04-22
}

one glucose molecule by oxidative phosphorylation, whereas the metabolism of glucose to lactate generates only 2 ATPs per molecule of glucose. This increased dependence on glycolysis for ATP production, instead of oxidative phosphorylation, in malignant cells was first reported by Dr Warburg, and is therefore known as the Warburg effect ${ }^{[4]}$. The metabolic shift to a higher rate of glycolysis can even be observed in patients with malignant tumors using positron emission tomography $(\mathrm{PET})^{[5]}$.

Beyond the current concept that the Warburg effect plays a central role in tumor metabolism, studies have shown that there may be dual metabolic (the Warburg effect and the nonglycolytic phenotype) natures in a given cell type, providing them with survival and growth advantages when adapting to a stressful microenvironment ${ }^{[6]}$. Cancer cells exhibit a glycolytic phenotype under regular conditions but can switch to a non-glycolytic phenotype under lactic acidosis. When tumor cells are cultured under conditions with sufficient glucose, they are initially glycolytic, and the resulting lactate generation leads to lactate accumulation and medium acidification, namely lactic acidosis. Lactic acidosis is an inevitable consequence of the Warburg effect and is common in most solid 
tumors $^{[7-10]}$. Lactic acidosis acidifies the cytosol and creates a low $\mathrm{pH}$ condition, thereby decreasing glycolytic flux and suppressing the activities of glycolytic enzymes, eventually impairing glycolysis. With a limited supply of glucose, tumor cells die quickly, but with lactate acidosis, they convert glucose at a slower rate and maintain cell proliferation by transitioning from the Warburg effect to a non-glycolytic phenotype ${ }^{[11]}$.

Recently, a new type of cancer metabolism, named the "Reverse Warburg effect" or "metabolic coupling," has been identified, and this effect can explain the contradictory phenomena in certain types of cancer cells with high mitochondrial respiration and low glycolysis rates. This concept implies a host-parasite relationship between cancer cells and glycolytic fibroblasts. In this model of two-compartment tumor metabolism, aerobic glycolysis occurs in stromal fibroblasts, and the produced recycled nutrients are transferred to adjacent tumor cells for supporting the fuel in the tricarboxylic acid cycle (TCA), thus producing abundant ATP and protecting them against apoptosis ${ }^{[12-14]}$. Therefore, this two-compartment tumor metabolism may contribute to chemoresistance or therapy failure in patients with cancer, making the disease difficult to cure using a single agent.

\section{The process of glycolysis}

Glycolysis is a process that takes place in the cytoplasm by converting glucose into pyruvate, lactate and hydrogen ions through nine enzymatic reaction steps, and there are several glycolytic enzymes that are involved in different steps of glycolysis and play important roles in the phenomenon of the Warburg effect ${ }^{[15]}$. In the first step, to keep the high rate of glucose consumption for aerobic glycolysis in tumor cells, the transport of glucose is a key factor. It has been found that the glucose transporters Glut-1 and sodium-glucose linked transporter 1 (SGLT1) are overexpressed in many cancer cells ${ }^{[16]}$. Intracellular glucose is phosphorylated into glucose6-phosphate by hexokinase (HK) in the first step, and then this product is converted into fructose-6-phosphate by glucose6-phosphate isomerase. In the third step, fructose-6-phosphate is phosphorylated into unstable fructose-1,6-biphosphate and relatively stable fructose-2,6-biphosphate, respectively, by phosphofructokinase-1 (PFK-1) and phosphofructokinase-2 (PFK-2), consuming one ATP molecule. The next step is the formation of glyceraldehyde-3-phosphate, which is converted into glycerate-1,3-diphosphate and then converted into 3-phosphoglycerate by phosphoglycerate kinase (PGK) with the generation of two ATP molecules. Next, 3-phosphoglycerate is isomerized into 2-phosphoglycerate by phosphoglyceratemutase (PGM), followed by phosphoenolpyruvate (PEP) formation. The last and rate-limiting step of glycolysis is catalyzed by pyruvate kinase (PK), converting PEP into pyruvate, and producing one ATP molecule at the same time. The $\mathrm{ATP} / \mathrm{AMP}$ ratio and intracellular $\mathrm{pH}$ have an important effect on PK activity ${ }^{[17]}$. There are four isoforms of pyruvate kinase: PKL, PKR, PKM1 and PKM2 $2^{[18]}$, among which the expression of PKM2 is upregulated in human tumor cells and cancerassociated fibroblasts ${ }^{[19,20]}$. PKM2 is present in two forms, including a dimer and tetramer, and its tetrameric form has higher glycolytic enzymatic activity than the dimeric form ${ }^{[21]}$. In addition, PKM2 can be translocated into the nucleus where it is involved in the regulation of gene transcription or functions as a protein kinase ${ }^{[22]}$. Contrary to PKM2, PKM1 seems to have a smaller effect on cell proliferation ${ }^{[23]}$. When tissues turn cancerous, only PKM2 is active ${ }^{[24]}$, and there is a decreased ratio of PKM1/PKM2 during malignant progression. The PKM1/PKM2 ratio is related to the cell cycle and tumor population density, thus switching PKM2 to PKM1. Changing this ratio may have some implications for cancer treatment. It has been observed that replacing PKM2 with PKM1 in human lung cancer cells can lead to inhibition of glycolysis and tumor xenograft formation in nude mice ${ }^{[25]}$. In the absence of oxygen, pyruvate is converted into lactate by lactate dehydrogenase in general.

\section{Molecular mechanisms of the Warburg effect}

A number of onco-proteins and tumor suppressors, including the PI3K/Akt/mTOR signaling pathway, Myc, hypoxiainducible factor and p53, have been reported to be involved in the regulation of this metabolic adaptation that favors tumor growth, cellular proliferation, angiogenesis and stress resistance (Figure 1).

\section{$\mathrm{PI}$ KK/Akt/mTOR signaling pathway}

The PI3K/Akt signaling pathway is involved in many cellular processes, such as inflammation, motility, autophagy and cancer progression. It has been observed that Akt causes a dose-dependent stimulation of glucose consumption and lactate production in human glioblastoma cells and malignant hematopoietic cells, and Akt-expressing cancer cells show an upregulated rate of glycolysis with no increase in total oxygen consumption, implying that the activation of Akt promotes cancer cell growth and survival by stimulating aerobic glycolysis $^{[26]}$. The activation of the Akt oncogene in cancer cells mobilizes glucose transporters to increase transportation of glucose. In addition, Akt can activate hexokinase 2 to enhance the glycolytic rate ${ }^{[27]}$.

The mTOR kinase exists in two structurally and functionally distinct multi-protein complexes, mTORC1 and mTORC2, and both complexes are critical regulators of cell metabolism, growth and proliferation. mTORC1 is a vital downstream effecter of PI3K/Akt that activates mTORC1 by inhibitory phosphorylation of the TSC1/2 complex, linking upstream signaling through Akt to protein translation, lipogenesis, cell proliferation, glycolysis and autophagy ${ }^{[28-30]}$. There are two main substrates of mTORC1: p70 ribosomal protein S6K kinase (S6K) and eIF4E binding protein (4EBP1). mTORC1 binds to eukaryotic initiation factor 3 (eIF3) and phosphorylates S6K under the condition of stimulation of growth signals, thus resulting in reinforced mRNA translation ${ }^{[31]}$. Active mTORC1 inhibits its downstream effecter 4EBP1 that restrains the protein translation initiation process by binding and inactivating eukaryotic translation initiation factor $4 \mathrm{E}$ (eIF4E), leading to the induction of GLUT1 and hexokinase 2, thereby increasing 


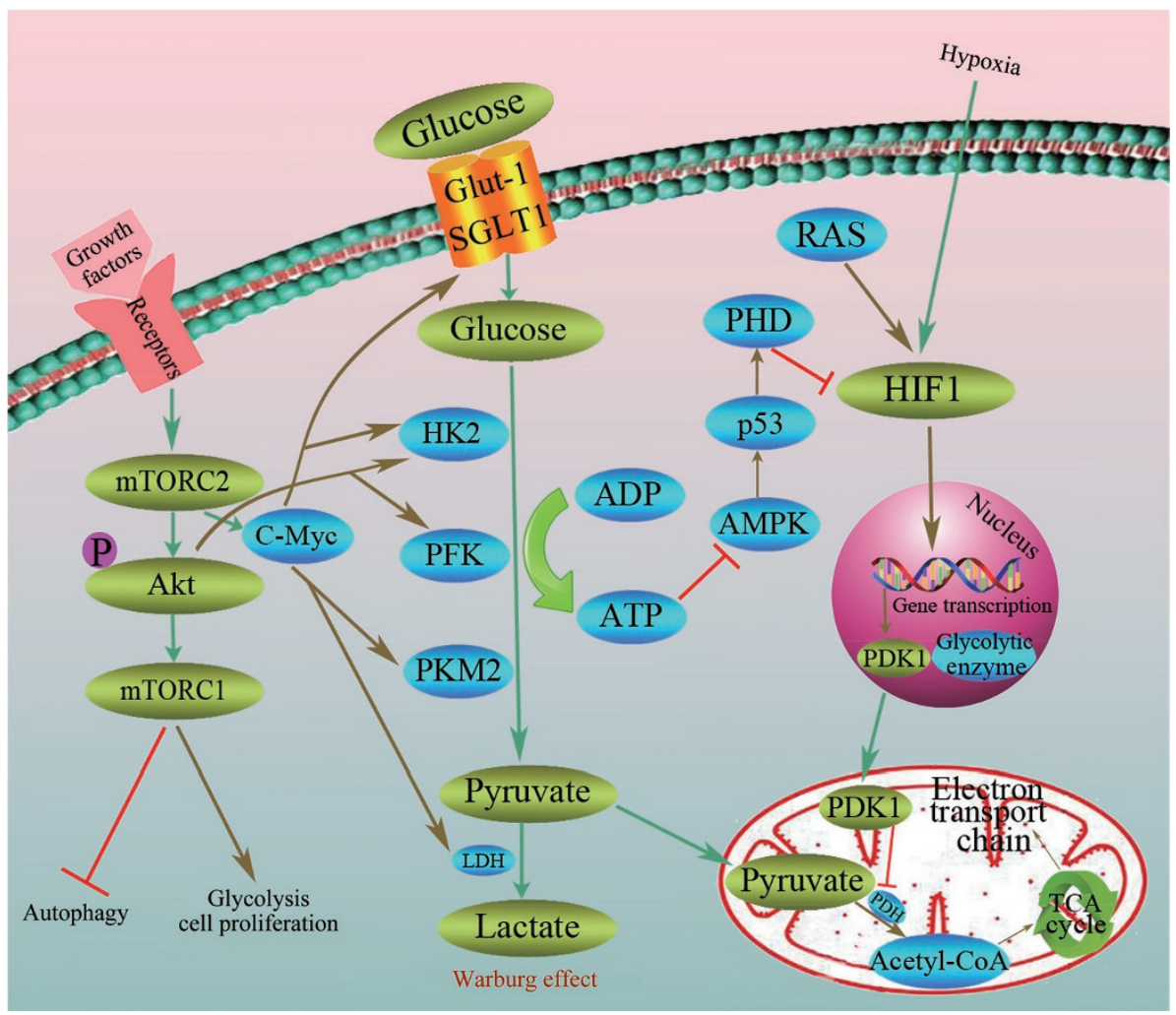

Figure 1. Molecular mechanisms of the Warburg effect. The Warburg effect, also termed "aerobic glycolysis," converts glucose into lactate by several enzymatic reaction steps even in the presence of oxygen, including glucose transporters Glut-1 and SGLT1, hexokinase (HK), phosphofructokinase (PFK) and PKM2. There are a number of onco-proteins and tumor suppressors, including PI3K/Akt/mTOR, Myc, hypoxia-inducible factor and p53, that are involved in the regulation of the Warburg effect.

glucose uptake and glycolysis ${ }^{[32]}$. It is generally believed that mTORC2 maximizes its activity primarily through phosphorylating Akt on serine 473, and controls the folding and stability of Akt protein, thus playing an important role in the Warburg effect $^{[33,34]}$. mTORC2 directly phosphorylates Akt on Ser473, thereby boosting the expression of the glucose transporter and activating HK2 as well as PFK-1, subsequently promoting glucose uptake and glycolysis ${ }^{[35,36]}$. A recent study has identified an Akt-independent signaling cascade by which mTORC2 upregulates the intracellular level of c-Myc, a key effecter of the Warburg effect, thus accelerating glycolysis in glioblastoma $(\mathrm{GBM})^{[37]}$.

\section{c-Myc}

c-Myc encoded by the Myc oncogene, a transcription factor controlling the process of cellular growth and metabolism, is overexpressed in the majority of human cancers, including colon, breast, bladder and prostate cancers ${ }^{[38]}$. The studies on transgenic animals have demonstrated that mice with overexpression of c-Myc in the liver had increased glycolytic enzyme activity and overproduction of lactic acid ${ }^{[39]}$, suggesting that c-Myc may play an important role in glycolysis. In addition, it has been found that activation of the c-Myc oncogene can upregulate many glucose metabolism enzyme genes, such as GLUT1, HK2, phosphofructokinase (PFKM), enolase 1 (ENO1), lactate dehydrogenase A (LDHA), and pyruvate dehydrogenase kinase (PDK1), thus stimulating the Warburg effect ${ }^{[40-43]}$. c-Myc is also linked to increased mitochondrial reactive oxygen species (ROS), which cause mitochondrial dysfunction and in turn help cancer cells to switch to glycolysis for their energy supply.

\section{Hypoxia-inducible factor-1 (HIF-1)}

HIFs, consisting of oxygen-labile a and constitutively expressed $\beta$ subunits, regulate diverse cellular processes such as erythropoiesis, angiogenesis, energy metabolism, ischemia, and inflammation via controlling the transcription of a variety of genes ${ }^{[44]}$. The stabilities of HIF-1a and HIF-2a are sensitive to the oxygen level and are regulated by HIF prolylhydroxylase (PHD), which facilitates the ubiquitin-mediated degradation of HIF in normoxic conditions. In normoxic human cells, HIF-1a is hydroxylated by PHD, and subsequently the prolylhydroxylated HIF-1a is degraded by $26 \mathrm{~S}$ proteasome ${ }^{[45]}$. Under hypoxic conditions, the HIF-1a protein accumulates due to the decreased prolylhydroxylation of HIF-1 ${ }^{[46]}$. Cancer cells show a stronger ability to adapt to a hypoxic environment than normal cells. Under hypoxic conditions, the HIF-1a is activated and involved in the regulation of glucose uptake and glycolysis in cancer cells via activating transcription of genes that encode glucose transporters and glycolytic enzymes. 
Evidence shows that pyruvate dehydrogenase kinase (PDK1), EGF receptor (EGFR) and HIF-1a are overexpressed in human glioblastoma multiforme (GBM) cells under hypoxia. As a direct target gene for HIF-1a, the upregulation of PDK1 may promote EGFR activation ${ }^{[4]]}$. Activation of NF-KB induced by EGFR promotes the expression of PKM2. Furthermore, PKM2 can be translocated into the nucleus as a result of the activation of EGFR in breast cancer cells, prostate cancer cells and glioblastoma cells, enhancing the expression of cyclin D1 as well as glycolytic enzyme and promoting cell proliferation and the Warburg effect ${ }^{[48-51]}$. In addition, HIF-1-mediated activation of PDK1 can also inhibit the activity of PDH, thereby impairing mitochondrial function and suppressing conversion of pyruvate to acetyl-CoA ${ }^{[52]}$.

\section{p53}

The tumor suppressor p53 can negatively regulate cell growth by transcriptionally inducing mTOR inhibitors such as IGFBP3, PTEN, TSC2, Sestrin1/2 and REDD1. p53 can reduce glycolysis by increasing the activity of fructose-2,6-bisphosphatase and inhibiting glucose transporters ${ }^{[53]}$. In addition, p53 is known to increase the process of oxidative phosphorylation via activating an element of the respiratory chain-like SCO2 gene $^{[54]}$. AMP-activated protein kinase (AMPK), a chief sensor of cellular energy, is inactivated when the intracellular AMP/ ATP ratio decreases. As a key target of AMPK, p53 is often mutated in human cancers, and either reduction or loss of p53 may be associated with the Warburg effect ${ }^{[55,56]}$.

\section{Anti-cancer therapy based on the Warburg effect}

Cancer cells show highly active aerobic glycolysis, and they really benefit from this metabolism. Therefore, it may be advisable to design anti-cancer drugs based on the characteristics and mechanisms of the aerobic glycolysis process and on the relationship between aerobic glycolysis and cancer progression.

As one of the hallmarks of cancer, aerobic glycolysis shows an increased rate of glucose consumption, and this high glucose uptake has been used in the clinic as a means of diagnosing and monitoring cancer treatment response by imaging uptake of $2-{ }^{18} \mathrm{~F}$-deoxyglucose with $\mathrm{PET}{ }^{[12]}$.

Because there are various critical enzymes involved in aerobic glycolysis, these enzymes may be exploited as targets for cancer therapies. Ritonavir, fasentin, genistein, STF-31 and WZB117 are anticancer drugs designed to target glucose transporter GLUT1 and exert anti-tumor effects by inhibiting glucose uptake in tumor cells, thus leading to cell death through glucose deprivation. As a key enzyme of aerobic glycolysis, HK is overexpressed in many malignant tumors. Several drugs have been exploited to inhibit HK such as 2-deoxy-Dglucose (2-DG), 3-BrPA and lonidamine ${ }^{[57,58]}$. Lonidamine, a derivative of indole, has anti-tumor activity via inhibiting HK2. This agent has now entered pre-clinical and clinical trials for treatment of cancer. The results of clinical trials have shown that lonidamine combined with chemotherapy, such as doxorubicin, had better therapeutic efficacy for the treatment of breast, prostate, melanoma, brain and ovarian tumors ${ }^{[59,60]}$. During the treatment process, there was drug-related liver toxicity. Therefore, there is some research work focusing on altering dosage forms or developing the targeted delivery systems of lonidamine to reduce its organ toxicity. 2-Deoxy$D$-glucose is a glucose analogue that can enter the cell and form 2-DG-P through phosphorylation. However, 2-DG-P is an "end" substance, because it cannot participate in further metabolic reactions, thereby blocking the glycolysis process. Studies have found that 2-deoxy- $D$-glucose in combination with PI3K or mTOR inhibitor PF-04691502 can shift aerobic glycolysis towards oxidative respiration in primary effusion lymphoma (PEL) cells, and this combination can cause strong cytotoxicity towards PEL cells but low toxicity to normal lymphocytes $^{[61]}$. 2-Deoxy-D-glucose has entered phase I clinical trials, and it is well tolerated and has less toxicity. Targeting PDK1 may be an attractive way to inhibit the growth and proliferation of GBM by shifting the Warburg phenotype to oxidative phosphorylation. As a unique PDK1 inhibitor, DCA lowered PDK1-EGFR activation, shifting the Warburg phenotype to oxidative phosphorylation (OXPHOS) in $\mathrm{GBM}^{[47]}$.

Another glycolytic enzyme, PFK-2, contains 4 isoenzymes: PFKFB1, 2, 3 and 4. PFKFB3 is expressed in many types of cancers, and targeting this enzyme has received extensive attention recently. Currently, the main PFKFB3 inhibitors include 3-(3-pyridinyl)-1-(4-pyridinyl)-2-propen-1-one (3PO) and 1-(4-pyridinyl)-3-(2-quinolinyl)-2-propen-1-one (PFK15). It was observed that $3 \mathrm{PO}$ could rapidly reduce glucose uptake, lactate secretion, and intracellular ATP in Jurkat T-cell leukemia cells ${ }^{[62]}$. PFK15, a synthesized derivative of 3PO, has antitumor activity through reducing ${ }^{18} \mathrm{FDG}$ uptake and F26BP content in xenografted tumors. In addition, this agent exhibits pro-apoptotic activity in transformed cells both in vitro and in tumors in vivo ${ }^{[63]}$.

As the final rate-limiting step enzyme in the glycolytic pathway, PKM2 plays a critical role in tumor cell metabolism and proliferation. Thus, developing novel anticancer drugs that target PKM2 may be of great importance. Thus far, several PKM2 inhibitors have been developed, including TT-232, VK3, VK5 and Compound 3. In contrast to Compound 3, which can also cause PKL inhibition ${ }^{[64]}$, VK3 and VK5 selectively inhibit PKM2 to reduce glycolysis in cancer cells. Recently, it has been found that oleanolic acid (OA), a natural compound distributed widely in plants, exerts its anticancer effect by inhibiting aerobic glycolysis through shifting PKM2 to PKM1 ${ }^{[65]}$. However, Israelsen et al recently reported that loss of PKM2 in a mouse model of breast cancer accelerates tumor formation, suggesting that pharmacological inhibition or therapeutic knockdown of PKM2 may not be effective standalone cancer therapies. The findings of this study highlighted the importance of understanding the context-dependent metabolic needs of cancer cells to effectively target metabolism for therapeutic benefit.

mTOR kinase inhibition was thought to be an effective anticancer approach. Rapamycin, an FDA-approved immunosuppression drug, and its analogues sirolimus, temsiroli- 
mus and everolimus are well-known allosteric inhibitors of mTORC1. These agents restrain mTORC1 activity but appear to have little effect on limiting mTORC2 activity. In preclinical studies, rapamycin and its analogues were reported to have antiproliferative activity in several types of cancers, and they are now being tested in the clinic for the treatment of lymphoma and neuroendocrine, endometrial and breast cancers. A recent study demonstrated that halofuginone $(\mathrm{HF})$ had a potent anticancer activity and slowed glycolysis via suppressing Akt/mTORC1 signaling and glucose metabolism. In HF-treated human colorectal cancer cells, there was a reduction in $\mathrm{mTORC1}$ and phosphorylation of Akt, causing a rapid decrease in HK2 and Glut1 ${ }^{[66]}$.

We recently found a novel role for eEF-2K, a negative regulator of protein synthesis, in promoting glycolysis and in tumor development and progression and the underlying molecular mechanism. We have reported that eEF-2K is overexpressed in several types of cancer, including breast cancer and glioma, and plays a crucial role in regulating activity of autophagy and level of cellular ATP in tumor cells. As there is evidence suggesting that autophagic activity and ATP production are closely linked to the rate of glycolysis, we queried whether eEF-2K has a role in this altered metabolic processes in tumor cells. We showed that deficiency in eEF-2K significantly reduced the uptake of glucose and decreased the production of lactate and ATP in tumor cells and in the Ras-transformed mouse embryonic fibroblasts (MEFs). In addition, depletion of eEF-2K reduced the ability of the transformed cells to proliferate and enhanced the sensitivity of tumor cells to chemotherapy both in vitro and in vivo. Our study not only reveals a novel regulator of glycolysis in cancer but also provides, to our knowledge, the first connection between the regulation of protein synthesis and glycolysis. These results also underscore the implication and importance of eEF-2K in cancer and the potential of this kinase as a novel target for prevention and treatment of this disease ${ }^{[67]}$.

In recent years, the concept of reversing the Warburg effect offers new perspectives to develop therapeutic strategies that focus on both of the targets, glycolysis and mitochondrial OXPHOS. Cancer cells can activate NFB and HIF-1a in adjacent stromal fibroblasts via oxidative stress promoted by ROS production, resulting in autophagy and mitophagy as well as glycolysis, which in turn can supply recycled nutrients to tumor cells for growth ${ }^{[6,55]}$. Therefore, it may be of significance to regulate glycolysis by targeting autophagy in the stroma. There are several FDA-approved candidate drugs targeting two-compartment tumor metabolism, including $N$-acetyl-cysteine (NAC), metformin, hydroxy-chloroquine and rapamycin or rapalogues. NAC, as an antioxidant to prevent oxidative stress, can block autophagy in the tumor stroma, thus interrupting the fuel supplied to the cell mitochondria. As a well-known antidiabetic drug, metformin is a direct AMPK activator, and it can reduce mitochondrial complex I activity, suggesting metformin may prevent cancer by inhibiting mitochondrial OXPHOS (Table 1).

In addition, it has been suggested that the reverse of the
Table 1. Several anti-cancer drugs based on targeting Warburg effect.

\begin{tabular}{ll}
\hline \multicolumn{1}{c}{ Drug(s) } & \multicolumn{1}{c}{ Target or mechanism } \\
\hline $\begin{array}{l}\text { Ritonavir, Fasentin, } \\
\text { STF-31, WZB117 }\end{array}$ & GLUTI inhibitor \\
2-DG, 3-BrPA, lonidamine & HK inhibitor \\
3PO, PFK15 & PFKFB3 inhibitor \\
OA, TT-232, VK3, VK5 & PKM2 inhibitor \\
Rapamycin & mTORC1 inhibitor \\
DCA & PDK1 inhibitor \\
NAC & Antioxidant, preventing oxidative stress \\
Hydroxy-chloroquine & Autophagy inhibitor \\
Metformin & OXPHOS inhibitor \\
Halofuginone & Akt/mTORC1 inhibitor \\
\hline
\end{tabular}

Warburg effect may be the basis of drug resistance. As stromal fibroblasts undergo aerobic glycolysis induced by cancer cells, they can protect adjacent tumor cells against apoptosis by providing nutrients to mitochondria in cancer cells to feed the TCA cycle. It was observed that MCF7 cells are sensitive to tamoxifen when cultured alone but become tamoxifenresistant when co-cultured with fibroblasts ${ }^{[68]}$. A drug combination has been developed to overcome such fibroblastinduced tamoxifen-resistance and shift cancer cells back to a glycolytic state. Dasatinib, a tyrosine kinase, in combination with tamoxifen, led to the death of MCF7 cells and reduced ROS production in both the stromal fibroblasts and MCF7 cancer cells ${ }^{[55]}$. This combination therapy (tamoxifen plus dasatinib) can overcome drug-resistance, has anti-oxidant effects, and may provide a novel approach to cancer treatment. For example, glycolysis inhibition combined with DNA damaging drugs or chemotherapeutic agents may be effective anticancer strategies through weakening cellular damage-repair capacity and enhancing drug cytotoxicity.

\section{Conclusion and expectations}

The Warburg effect involves complex control of the expression of multiple genes and pathways, and modulating one target or segment may not be sufficient to suppress tumors and might even result in drug resistance. Therefore, it is worth our attention to explore combination therapy aimed at multiple targets. With the further study of aerobic glycolysis in cancer cells, it can be anticipated that an increasing number of novel therapeutic agents or preventive strategies will be developed. Additionally, it can also be regarded as efficacious to inhibit tumor proliferation and invasion by relieving the Warburg effect via invigorating blood circulation and improving oxygen supply when there is a glucose shortage.

\section{Acknowledgements}

This work was supported by grants from the National Basic Research Program of China (973 Program, 2015CB910700) (Yan CHENG) and the National Natural Science Foundation of China (81422051, 81472593, and 31401208) (Yan CHENG). 


\section{References}

1 DeBerardinis RJ, Lum JJ, Hatzivassiliou G, Thompson CB. The biology of cancer: metabolic reprogramming fuels cell growth and proliferation. Cell Metab 2008; 7: 11-20.

2 Vander Heiden MG, Cantley LC, Thompson CB. Understanding the Warburg effect: the metabolic requirements of cell proliferation. Science 2009; 324: 1029-33.

3 Warburg 0. On the origin of cancer cells. Science 1956; 123: 30914.

4 Warburg O, Posener KEN. Über den stoffwechsel der carcinomzelle. Biochem Zeitschr 1924; 152: 309-44.

5 Weiler-Sagie M, Bushelev O, Epelbaum R, Dann EJ, Haim N, Avivi I, et al. ${ }^{18} \mathrm{~F}-\mathrm{FDG}$ avidity in lymphoma readdressed: a study of 766 patients. J Nucl Med 2010; 51: 25-30.

6 Xie J, Wu H, Dai C, Pan Q, Ding Z, Hu D, et al. Beyond Warburg effectdual metabolic nature of cancer cells. Sci Rep 2014; 4: 4927.

7 Gatenby RA, Gillies RJ. Why do cancers have high aerobic glycolysis? Nat Rev Cancer 2004; 4: 891-9.

8 Gallagher FA, Kettunen MI, Day SE, Hu DE, Ardenkfaer-Larsen JH, Zandt $\mathrm{Rl}$, et al. Magnetic resonance imaging of $\mathrm{pH}$ in vivo using hyperpolarized ${ }^{13} \mathrm{C}$-labelled bicarbonate. Nature 2008; 453: 940-3.

9 Thistlethwaite AJ, Leeper DB, Moylan DJ 3rd, Nerlinger RE. pH distribution in human tumors. Int J Radiat Oncol Biol Phys 1985; 11 : 1647-52.

10 van Sluis R, Bhulwalla ZM, Raohunand N, Ballesteros P, Alvarez J, Cerdan $\mathrm{S}$, et al. In vivo imaging of extracellular pH using $1 \mathrm{HMRSI}$. Magn Reson Med 1999; 41: 743-50.

11 Wu H, Ding Z, Hu D, Sun F, Dai C, Xie J, et al. Central role of lactic acidosis in cancer cell resistance to glucose deprivation-induced cell death. J Pathol 2012; 227: 189-99.

12 Sotgia F, Martinez-Outschoorn UE, Pavlides S, Howell A, Pestell RG, MP L. Understanding the Warburg effect and the prognostic value of stromal caveolin-1 as a marker of a lethal tumor microenvironment. Breast Cancer Res 2011; 13: 213.

13 Pavlides S, Whitaker-Menezes D, Castello-Cros R, Flomenberg N, Witkiewicz AK, Frank PG, et al. The reverse Warburg effect: aerobic glycolysis in cancer associated fibroblasts and the tumor stroma. Cell Cycle 2009; 8: 3984-4001.

14 Vincent AS, Phan TT, Mukhopadhyay A, Lim HY, Halliwell B, Wong KP. Human skin keloid fibroblasts display bioenergetics of cancer cells. J Invest Dermatol 2008; 128: 702-9.

15 Lane AN, Fan TW, Higashi RM. Metabolic acidosis and the importance of balanced equations. Metabolomics 2008; 5: 163-5.

16 Casneuf VF, Casneuf VF, Fonteyne P, Van Damme N, Demetter P, Pauwels $\mathrm{P}$, et al. Expression of SGLT1, Bcl-2 and p53 in primary pancreatic cancer related to survival. Cancer Invest 2008; 26: 8529.

17 Kinderlerer J, Ainsworth S, Morris CN, Rhodes N. The regulatory properties of yeast pyruvate kinase. Effect of pH. Biochem J 1986; 234: $699-703$.

18 Imamura K, Tanaka T. Multimolecular forms of pyruvate kinase from rat and other mammalian tissues. I. Electrophoretic studies. J Biochem 1972; 71: 1043-51.

19 Luo W, Semenza GL. Emerging roles of PKM2 in cell metabolism and cancer progression. Trends Endocrinol Metab 2012; 23: 560-6.

20 Bonuccelli G, Whitaker-Menezes D, Castello-Cros R, Pavlides S, Pestell RG, Fatatis A, et al. The reverse Warburg effect: glycolysis inhibitors prevent the tumor promoting effects of caveolin-1 deficient cancer associated fibroblasts. Cell Cycle 2010; 9: 1960-71.

21 Mazurek S, Boschek CB, Hugo F, Eigenbrodt E. Pyruvate kinase type M2 and its role in tumor growth and spreading. Semin Cancer Biol

2005; 15: 300-8.

22 Yang W, Lu Z. Nuclear PKM2 regulates the Warburg effect. Cell Cycle 2013; 12: 3154-8.

23 Barger JF, Plas DR. Balancing biosynthesis and bioenergetics: metabolic programs in oncogenesis. Endocr Relat Cancer 2010; 17: R287-304.

24 Christofk HR, Vander Heiden MG, Wu N, Asara JM, Cantley LC. Pyruvate kinase $\mathrm{M} 2$ is a phosphotyrosine binding protein. Nature 2008; 452: 181-6.

25 Christofk HR, Vander HM, Harris MH, Ramanathan A, Gerszten RE, Wei R, et al. The M2 splice isoform of pyruvate kinase is important for cancer metabolism and tumour growth. Nature 2008; 452: 230-3.

26 Elstrom RL, Bauer DE, Buzzai M, Karnauskas R, Harris MH, Plas DR, et al. Akt stimulates aerobic glycolysis in cancer cells. Cancer Res 2004; 64: 3892-9.

27 Gottlob K, Majewski N, Kennedy S, Kandel E, Robey RB, Hay N. Inhibition of early apoptotic events by Akt/PKB is dependent on the first committed step of glycolysis and mitochondrial hexokinase. Genes Dev 2001; 15: 1406-18.

28 Nazio F, Strappazzon F, Antonioli M, Bielli P, Cianfanelli V, Bordi M, et al. mTOR inhibits autophagy by controlling ULK1 ubiquitylation, selfassociation and function through AMBRA1 and TRAF6. Nat Cell Biol 2013; 15: 406-16.

29 Ben-Sahra I, Howell JJ, Asara JM, Manning BD. Stimulation of de novo pyrimidine synthesis by growth signaling through mTOR and S6K1. Science 2013; 339: 1323-8.

30 Zhang Y, Nicholatos J, Dreier JR, Ricoult SJ, Widenmaier SB, Hotamisligil GS, et al. Coordinated regulation of protein synthesis and degradation by mTORC1. Nature 2014; 513: 440-3.

31 Holz MK, Blenis J. Identification of S6 kinase 1 as a novel mammalian target of rapamycin (mTOR)-phosphorylating kinase. J Biol Chem 2005; 28: 26089-93.

32 Maiese K, Chong ZZ, Shang YC, Wang S. mTOR: ontarget for novel therapeutic strategies in the nervous system. Trends Mol Med 2013; 19: 51-60.

33 Sarbassov DD, Guertin DA, Ali SM, Sabatini DM. Phosphorylation and regulation of Akt/PKB by the rictor-mTOR complex. Science 2005; 307: 1098-101.

34 Facchinetti V, Ouyang W, Wei H, Soto N, Lazorchak A, Gould C, et al. The mammalian target of rapamycin complex 2 controls folding and stability of Akt and protein kinase C. Embo J 2008; 27: 1932-43.

35 Gottlob K, Majewski N, Kennedy S, Kandel E, Robey RB, Hay N. Inhibition of early apoptotic events by Akt/PKB is dependent on the first committed step of glycolysis and mitochondrial hexokinase. Genes Dev 2001; 15: 1406-18.

36 Kohn AD, Summers SA, Birnbaum MJ, Roth RA. Expression of a constitutively active Akt Ser/Thr kinase in 3T3-L1 adipocytes stimulates glucose uptake and glucose transporter 4 translocation. J Biol Chem 1996; 271: 31372-8.

37 Masui K, Tanaka K, Akhavan D, Babic I, Gini B, Matsutani T, et al. mTOR complex 2 controls glycolytic metabolism in glioblastoma through FoxO acetylation and upregulation of c-Myc. Cell Metab 2013; 18: 726-39.

38 Dang CV, Le A, Gao P. MYC-induced cancer cell energy metabolism and therapeutic opportunities. Clin Cancer Res 2009; 15: 6479-83.

39 Valera A, Pujol A, Gregori X, Riu E, Visa J, Bosch F. Evidence from transgenic mice that myc regulates hepatic glycolysis. Faseb J 1995; 9: $1067-78$.

40 Lewis BC, Shim H, Li Q, Wu CS, Lee LA, Maity A, et al. Identification of putative c-Myc-responsive genes: characterization of rcl, a novel growth-related gene. Mol Cell 1997; 17: 4967-78. 
41 Shim H, Dolde C, Lewis BC, Wu CS, Dang G, Jungmann RA, et al. C-Myc transactivation of LDH-A: implications for tumor metabolism and growth. Proc Natl Acad Sci U S A 1997; 94: 6658-63.

42 Kim JW, Gao P, Liu YC, Semenza GL, Dang CV. Hypoxia-inducible factor 1 and dysregulated c-Myc cooperatively induce vascular endothelial growth factor and metabolic switches hexokinase 2 and pyruvate dehydrogenase kinase 1. Mol Cell Biol 2007; 27: 7381-93.

43 Osthus RC, Shim H, Kim S, Li Q, Reddy R, Mukherjee M, et al. Deregulation of glucose transporter 1 and glycolytic gene expression by c-Myc. J Biol Chem 2000; 275: 21797-800.

44 Liao SH, Zhao XY, Han YH, Zhang J, Wang LS, Xia L, et al. Proteomicsbased identification of two novel direct targets of hypoxia-inducible factor-1 and their potential roles in migration/invasion of cancer cells.. Proteomics 2009; 9: 3901-12.

45 Maxwell PH, Wiesener MS, Chang GW, Clifford SC, Vaux EC, Cockman $\mathrm{ME}$, et al. The tumour suppressor protein $\mathrm{VHL}$ targets hypoxiainducible factors for oxygen-dependent proteolysis. Nature 1999; 399: 271-5.

46 Kaelin WJ, Ratcliffe PJ. Oxygen sensing by metazoans: the central role of the HIF hydroxylase pathway. Mol Cell 2008; 30: 393-402.

47 Velpula KK, Bhasin A, Asuthkar S, Tsung AJ. Combined targeting of PDK1 and EGFR triggers regression of glioblastoma by reversing the Warburg effect. Cancer Res 2013; 73: 7277-89.

48 Stetak A, Veress R, Ovadi J, Csermely P, Keri G, Ullrich A. Nuclear translocation of the tumor marker pyruvate kinase $\mathrm{M} 2$ induces programmed cell death. Cancer Res 2007; 67: 1602-8.

49 Yang W, Xia Y, Ji H, Zheng Y, Liang J, Huang W, et al. Nuclear PKM2 regulates $\beta$-catenin transactivation upon EGFR activation. Nature 2011; 480: 118-22.

50 Yang W, Zheng Y, Xia Y, Ji H, Chen X, Guo F, et al. ERK1/2-dependent phosphorylation and nuclear translocation of PKM2 promotes the Warburg effect. Nat Cell Biol 2012; 14: 1295-304.

51 Yang W, Xia Y, Hawke D, Li X, Liang J, Xing D, et al. PKM2 phosphorylates histone $\mathrm{H} 3$ and promotes gene transcription and tumorigenesis. Cell 2012; 150: 685-96.

52 Kim JW, Tchernyshyov I, Semenza GL, Dang CV. HIF-1-mediated expression of pyruvate dehydrogenase kinase: a metabolic switch required for cellular adaptation to hypoxia. Cell Metab 2006; 3: 17785.

53 Feng Z, Levine AJ. The regulation of energy metabolism and the IGF1/mTOR pathways by the p53 protein. Trends Cell Biol 2010; 20 : 427-34.

54 Bensinger SJ, HR C. New aspects of the Warburg effect in cancer cell biology. Semin Cell Dev Biol 2012; 23: 352-61.

55 Gonzalez CD, Alvarez S, Ropolo A, Rosenzvit C, Bagnes MF, Vaccaro
MI. Autophagy, Warburg, and Warburg reverse effects in human cancer. Biomed Res Int 2014; 2014: 926729.

56 Kim JW, Dang CV. Cancer's molecular sweet tooth and the Warburg effect. Cancer Res 2006; 66: 8927-30.

57 Weber G, Cantero A. Glucose-6-phosphatase activity in normal, precancerous, and neoplastic tissues. Cancer Res 1955; 15: 105-8.

58 Hadjiolov D. Glucose-6-phosphatase activity in primary rat liver tumors induced by high doses of 4-dimethylaminoazo-benzene. Zeit für Krebs 1969; 72: 43-6.

59 Amadori D, Frassineti GL, De Matteis A, Mustacchi G, Santoro A, Cariello $S$, et al. Modulating effect of lonidamine on response to doxorubicin in metastatic breast cancer patients: results from a multicenter prospective randomized trial. Breast Cancer Res Treat 1998; 49: 209-17.

60 Nath K, Nelson DS, Heitjan DF, Leeper DB, Zhou R, Glickson JD. Lonidamine induces intracellular tumor acidification and ATP depletion in breast, prostate and ovarian cancer xenografts and potentiates response to doxorubicin. Nmr Biomed 2015; 28: 281-90.

61 Mediani L, Gibellini F, Bertacchini J, Frasson C, Bosco R, Accordi B, et al. Reversal of the glycolytic phenotype of primary effusion lymphoma cells by combined targeting of cellular metabolism and PI3K/Akt/ mTOR signaling. Oncotarget 2015; 7: 5521-37.

62 Clem B, Telang S, Clem A, Yalcin A, Meier J, Simmons A, et al. Smallmolecule inhibition of 6-phosphofructo-2-kinase activity suppresses glycolytic flux and tumor growth. Mol Cancer Ther 2008; 7: 110-20.

63 Clem BF, O'Neal J, Tapolsky G, Clem AL, Imbert-Fernandez Y, Kerr DN, et al. Targeting 6-phosphofructo-2-kinase (PFKFB3) as a therapeutic strategy against cancer. Mol Cancer Ther 2013; 12: 1461-70.

64 Vander Heiden MG, Christofk HR, Schuman E, Subtelny AO, Sharfi H, Harlow EE, et al. Identification of small molecule inhibitors of pyruvate kinase M2. Biochem Pharmacol 2010; 79: 1118-24.

65 Liu J, Wu N, Ma L, Liu M, Liu G, Zhang Y, et al. Oleanolic acid suppresses aerobic glycolysis in cancer cells by switching pyruvate kinase type M isoforms. PLoS One 2014; 9: e91606.

66 Chen GQ, Tang CF, Shi XK, Lin CY, Fatima S, Pan XH, et al. Halofuginone inhibits colorectal cancer growth through suppression of Akt/mTORC1 signaling and glucose metabolism. Oncotarget 2015; 6: 24148-62.

67 Cheng Y, Ren X, Yuan Y, Shan Y, Li L, Chen X, et al. eEF-2 kinase is a critical regulator of Warburg effect through controlling PP2A-A synthesis. Oncogene 2016 May 16. doi: 10.1038/onc.2016.166.

68 Martinez-Outschoorn UE, Lin Z, Ko YH, Goldberg AF, Flomenberg N, Wang $\mathrm{C}$, et al. Understanding the metabolic basis of drug resistance: therapeutic induction of the Warburg effect kills cancer cells. Cell Cycle 2011; 10: 2521-8. 